[ Commentary on Cecelia Heyes (2018). Cognitive Gadgets. Harvard University Press.

Published as: Baggs, E., Raja, V., \& Anderson, M. (2019). Culture in the world shapes culture in the head (and vice versa). Behavioral and Brain Sciences, 42, E172

https://doi.org/10.1017/S0140525X19001079 ]

\title{
Culture in the world shapes culture in the head (and vice versa)
}

Edward Baggs, ${ }^{a}$ Vicente Raja, ${ }^{\mathrm{b}}$ and Michael L. Anderson ${ }^{\mathrm{c}}$

${ }^{a}$ Bartlett School of Architecture, University College London, London WC1H OQB, United Kingdom; ${ }^{b}$ The Rotman Institute of Philosophy, Western University, London, ON N6A 5B7, Canada; ${ }^{\circ}$ The Rotman Institute of Philosophy, Western University, London, ON N6A 5B7, Canada.

\section{ed.baggs@gmail.com \\ vgalian@uwo.ca \\ mande54@uwo.ca}

\begin{abstract}
We agree with Heyes that an explanation of human uniqueness must appeal to cultural evolution, and not just genes. Her account, though, focuses narrowly on internal cognitive mechanisms. This causes her to mischaracterize human behavior, and to overlook the role of material culture. A more powerful account would view cognitive gadgets as spanning organisms and their (shared) environments.
\end{abstract}




\section{Text}

We are pleased for the opportunity to engage with such an interesting and well-presented account of the historical origins and physical bases of distinctly human cognitive capacities. We especially applaud Heyes' willingness to challenge deep intuitions about the role of genetic inheritance. It has too often been assumed that an explanation of our uniqueness must ultimately be cashed out in terms of genes. But, as Heyes (2018) repeatedly shows, there is scant evidence that genetic adaptations alone can account for uniquely human behaviors such as social imitation or language. We agree that cultural learning is an important force shaping these aspects of human cognition. Where we disagree is with the methodological assumption that good cognitive science focuses exclusively on the internal (neural) processes that shape behavior. In fact, cognition relies on extensive and mutual interactions between organism and environment. The environment should not be left out of the explanation. In what follows, we will try to show how a core distinction that drives Heyes' account, between grist and mills, potentially blinds her to the importance of environmental contributions to cognition, culture, and learning. We believe that giving up on a strict distinction between grist and mill can lead to a theory of the origins and bases of human cognition largely consistent with the cognitive gadgets hypothesis, but with broader theoretical and explanatory reach. 
Early in Cognitive Gadgets, Heyes (2018) writes: "The cognitive gadgets answer [to what makes humans distinct] is concerned not with the grist of the mind - what we do and make - but with its mills, the way the mind works ...” (p. 14). In an earlier paper, Heyes illustrated this distinction by appealing to reading - her paradigm case of a cognitive gadget (Heyes 2012a). In reading, the "ideas and values coded" in the text are grist, whereas the neurocognitive pathways that have developed in a literate human, and that enable her to understand the text, are mills. On the face of it, this seems like a neat distinction. It is certainly a reasonable one to make if your goal is to challenge the nativist assumption that distinctively human traits must be explained in terms of a genetic endowment. The distinction allows Heyes to make a clear negative argument against this assumption. Her argument is roughly: culture partly shapes cognitive mills; therefore, genes alone are insufficient to explain human uniqueness.

It is clear, however, that the gadget theory is intended to be more than just a negative challenge to the instinct theory. It also aims to be a positive framework for future evolutionary thinking about cognition (Heyes 2018, p. 77). From this point of view, we suggest that the strict distinction between grist and mills is problematic. It overlooks the ways that grist - the things we do and make - can also play a role in cognitive processes. Leaving grist out of the equation leads to an unnecessarily narrow, and individualistic, account of what human intelligence is. The point here is not that Heyes' book is too narrow in scope, and therefore fails to offer a complete account of "the cultural evolution of thinking." Who could possibly do that? It is rather that by being too narrow in scope it distorts the nature of the cognitive mechanisms she hopes to describe. 
It has long been a staple of cognitive science that some actions are special. They are not just the product of thinking, but they produce and enhance thinking. Simple epistemic actions such as rotating a jigsaw puzzle piece to better perceive fit (Kirsh \& Maglio 1994), or writing down the intermediate steps in a long-division problem to reduce memory load (Clark \& Chalmers 1998), or gesturing over written equations, or in conversation (Goldin-Meadow 2005) are not just the product of thinking but part of the cognitive processing. Similarly, some tools are so integral to behavior that we experience them as part of our body. The blind man does not feel with his hand the movements of the cane; he feels the world at the end of the cane (Merleau-Ponty 2013). And it has long been known - and has recently become big business in office design! - that the nature of one's physical environs changes how one thinks, behaves, and interacts (Kirsh 2005). These claims are not wholly uncontroversial, but the examples could be multiplied manyfold (Arendash et al. 2004; Barth \& Funke 2010; Belland et al. 2013; Clark 1998; Flick 2000), and each seems to represent a (possible) counterexample to a strict grist-mill distinction. Grist is sometimes mill, or an important component in a cognitive mill, and ignoring this distorts the nature of human cognitive gadgets. Not incidentally, Heyes herself insists that cognitive mills are themselves the product of human behavior - social/cultural learning in particular - so it's also the case that mills are grist. The distinction, compelling at first glance, seems to fall apart under close scrutiny.

Why does it matter that the distinction is blurrier than it might initially seem? Because her commitment to the distinction causes Heyes to miss important points that we 
suspect she actually agrees with. Specifically, it leads her to overlook the ways that we reshape the world itself in ways that facilitate, constrain, and structure the cognitive work that we do. This idea is key to modern biological thinking. At the root of the extended evolutionary synthesis (Laland et al. 2015) is the notion of organism-environment mutuality, and in particular the concept of niche construction: the idea that animals reshape their environments through their actions, and this in turn structures the selection pressures exerted on current and future generations (Laland et al. 2000; Lewontin 1983). (Heyes does mention niche construction once in the book, on p. 9, in a list of ideas that she promises to return to later.)

It would not be an exaggeration to say that human evolution does not make sense except in the light of niche construction. Human evolution is characterized by a progressive expansion of the ways in which we have changed our environment, from the use of primitive tools through the reshaping of land and livestock with agriculture to our modern predicament in which we have upset the self-regulating balance of the climate itself. On a broad reading of the word, all of this is cultural. It is the result of our use of and engagement with material culture. Cognitive Gadgets uses a more narrow definition of culture. Heyes presents cultural evolution essentially as a process by which ideas are transmitted from one (cultural) generation to the next. Culture is thus thought of as something that is carried around inside the heads of individual actors. But material culture does not fit this model. The architecture of Venice is material culture. It has been there for hundreds of years. But it is not carried around inside the people in Venice. It is persisting, worldly structure. We could claim, metaphorically, that the structure of Venice is 
"transmitted" from one generation to the next. But this does not capture the fact that Venice is a city that has its own continuing existence as a set of buildings and practices and behavior settings. The place itself structures the behavior of the people in it. Books, paintings, tools, cities: All of these are aspects of culture that do not have to be transmitted, but reside in the shared environment of a community.

The focus on cognitive mills leads Heyes to mischaracterize the role of the environment in a more basic way still. The closest she comes to recognizing the causal role of the environment in action is in a brief discussion of joint actions such as "moving furniture or dancing together" (p. 164). Heyes is, we think rightly, resistant towards mindreading accounts of such actions according to which each party to the action mentally represents the other's beliefs. She points out that the fact that we are able to attend to the same thing in the environment is often sufficient to account for our having the same belief as one another - for example, that "there's a puddle on the floor" (p. 164). This, though, leaves intact the assumption that our actions must be explained with reference to our individual beliefs. Now suppose that you and I dance around the edge of the puddle, carefully avoiding getting our shoes wet. Then I jump in the puddle, splashing you. Immature, perhaps. But there was a point to it. The point was to demonstrate that the puddle itself is a component in our action control. The whole sequence of seeing the puddle, avoiding it, jumping in it, is possible because the puddle is actually there, and is meaningful to us because a puddle is the kind of thing our bodies can interact with. We weren't dancing around our beliefs about the puddle, and it wasn't your belief about the puddle that caused you to get wet. 
As it is with puddles, so it is with highly developed forms of craftsmanship. Acquiring the skills and dexterity to produce traditional stone artifacts such as axe heads would have taken many years of practice. In modern-day populations where such technologies are still produced, it can take 10 years for a learner to master the skill fully, under instruction from a more expert producer (Stout 2002). The point here is that the whole process of learning involves engagement with the stone itself, in a social context; the practice never becomes divorced from the material.

Maintaining the grist-mill distinction distorts Heyes' thinking in some more subtle ways, too. For instance, she characterizes "turnstile learning" as asocial. One confronts a turnstile (or other artifact) and by trial-and-error figures out its operation (p. 86). But that's not quite right. Turnstiles and other artifacts use design conventions to guide perception and behavior, conventions which are themselves reflected in and preserved by the built environment. For humans, there is perhaps very little learning that is truly asocial, even when it doesn't involve synchronic interactions with another human being.

Our final point is about group cognition. Heyes allows that cognitive gadgets are the result of multilevel selection. In the penultimate sentence of the book, she asserts: "Distinctively human cognitive processes are products of cultural group selection" (2018, p. 223). Group selection is anathema to selfish gene theorists. But despite her willingness to adopt this particular heresy, Heyes remains committed to methodological individualism for explanations of cognitive phenomena. We think this is a missed opportunity. 
Can there be group gadgets? There are good reasons to explore how the gadget concept might be applied to groups. One of the distinctive features of human societies is the division of labor among members of the population. One could argue that the division of labor is itself a cognitive phenomenon. It expands the possibilities for human thinking because it allows the group to solve problems across an expanding range of activities. Heyes appears to be sympathetic to some version of group intelligence. The specific form of group selection theory that she appeals to is the collective intelligence hypothesis, according to which cumulative culture depends on a population maintaining a minimum group size sufficient to ensure that culturally evolved practices are kept alive (Henrich 2015). A more powerful formulation of the gadget theory would, we suggest, leave open the possibility that an explanation of the mechanisms of cumulative culture needs to appeal not only to processes within the individual, but also to interpersonal processes that structure and constrain how practices are maintained by a population.

If Heyes' goal is only to provide a competitor to the instinct theory, then we think she has succeeded admirably. Allowing that cognitive mills can have their origins partly in culture is an improvement on insisting on an exclusively genetic explanation. The gristmill distinction, though, places an unnecessary limit on what this new theory can achieve. We think a more powerful approach, and one that will allow the gadget theory to serve more effectively as a framework for future research, is to abandon the distinction in favor of a mutualistic understanding of organisms and their environments. Culture is not just in the head. 


\section{ACKNOWLEDGMENTS}

EB was supported by funding from the European Union's Horizon 2020 research and innovation programme under grant agreement No 706432. MLA is supported by a Canada Research Chair funded by SSHRC.

References[Edward Baggs, Vicente Raja, and Michael L. Anderson][EB]

Arendash, G. W., Garcia, M. F., Costa, D. A., Cracchiolo, J. R., Wefes, I. M., \& Potter, H. (2004). Environmental enrichment improves cognition in aged Alzheimer's transgenic mice despite stable $\beta$-amyloid deposition. Neuroreport, 15(11), 17511754.

Barth, C. M., \& Funke, J. (2010). Negative affective environments improve complex solving performance. Cognition and Emotion, 24(7), 1259-1268.

Belland, B. R., Kim, C., \& Hannafin, M. J. (2013). A framework for designing scaffolds that improve motivation and cognition. Educational Psychologist, 48(4), 243-270.

Clark, A. (1998). Being there: Putting brain, body, and world together again. MIT Press.

Clark, A., \& Chalmers, D. (1998). The extended mind. Analysis, 58(1), 7-19.

Flick, L. B. (2000). Cognitive scaffolding that fosters scientific inquiry in middle level science. Journal of Science Teacher Education, 11(2), 109-129.

Goldin-Meadow, S. (2005). Hearing gesture: How our hands help us think. Harvard University Press.

Henrich, J. (2015). The secret of our success: How culture is driving human evolution, domesticating our species, and making us smarter. Princeton University Press. 
Heyes, C. (2018) Cognitive gadgets. The cultural evolution of thinking. Harvard University Press.

Heyes, C. (2012a) Grist and mills: on the cultural origins of cultural learning. Philosophical Transactions of the Royal Society B: Biological Sciences, 367(1599):2181-2191.

Kirsh, D. (2005). Metacognition, distributed cognition and visual design. In: Cognition, education, and communication technology, ed. P. Gardenfors and P. Johansson, pp. 147-180. Routledge.

Kirsh, D., \& Maglio, P. (1994). On distinguishing epistemic from pragmatic action. Cognitive Science, 18(4), 513-549.

Laland, K. N., Odling-Smee, J., and Feldman, M. W. (2000). Niche construction, biological evolution, and cultural change. Behavioral and Brain Sciences, 23(1):131-146.

Laland, K. N., Uller, T., Feldman, M. W., Sterelny, K., Müller, G. B., Moczek, A., Jablonka, E., and Odling- Smee, J. (2015). The extended evolutionary synthesis: its structure, assumptions and predictions. Proceedings of the Royal Society B: Biological Sciences, 282(1813):20151019.

Lewontin, R. C. (1983). The organism as the subject and object of evolution. Scientia, 118(1-8):65-95.

Merleau-Ponty, M. (2013). Phenomenology of perception. Routledge.

Stout, D. (2002). Skill and cognition in stone tool production: An ethnographic case study from Irian Jaya. Current Anthropology, 43(5): 693-722. 